\title{
Double-blind, randomised, placebo-controlled intervention trial to evaluate the effects of Bifidobacterium longum CECT 7347 in children with newly diagnosed coeliac disease
}

\author{
Marta Olivares $^{1}$, Gemma Castillejo ${ }^{2}$, Vicente Varea ${ }^{3}$ and Yolanda Sanz ${ }^{1 *}$ \\ ${ }^{1}$ Microbial Ecology, Nutrition and Health Research Group, Institute of Agrochemistry and Food Technology, \\ National Research Council (IATA-CSIC), Avenida Agustín Escardino, 7, Paterna-Valencia 46980, Spain \\ ${ }^{2}$ Paediatric Gastroenterology Unit, Hospital Universitari Sant Joan de Reus, Universitat Rovira i Virgili, Tarragona, Spain \\ ${ }^{3}$ Division of Paediatric Gastroenterology, Nutrition and Hepatology, Hospital Universitario Sant Joan de Deu, \\ Barcelona, Spain
}

(Submitted 14 August 2013 - Final revision received 11 February 2014 - Accepted 17 February 2014 - First published online 28 April 2014)

\begin{abstract}
Interactions between the immune system and the intestinal microbiota may play a role in coeliac disease (CD). In the present study, the potential effects of Bifidobacterium longum CECT 7347 in children with newly diagnosed CD were evaluated. A double-blind, randomised, placebo-controlled trial was conducted in thirty-three children who received a capsule containing either B. longum CECT 7347 (10 $0^{9}$ colonyforming units) or placebo (excipients) daily for 3 months together with a gluten-free diet (GFD). Outcome measures (baseline and postintervention) included immune phenotype of peripheral blood cells, serum cytokine concentration, faecal secretory IgA (sIgA) content, anthropometric parameters and intestinal microbiota composition. Comparisons between the groups revealed greater height percentile increases $(P=0.048)$ in the B. longum CECT 7347 group than in the placebo group, as well as decreased peripheral CD $3^{+} \mathrm{T}_{\text {lymphocytes }}$ $(P=0.004)$ and slightly reduced TNF- $\alpha$ concentration $(P=0.067)$. Within-group comparisons of baseline and final values did not reveal any differences in T lymphocytes and cytokines in the placebo group, while decreased $\mathrm{CD}^{+}(P=0.013)$ and human leucocyte antigen (HLA)$\mathrm{DR}^{+} \mathrm{T}$ lymphocytes $(P=0.029)$ and slightly reduced TNF- $\alpha$ concentration $(P=0.085)$ were detected in the B. longum CECT 7347 group. Comparison between the groups showed that the administration of B. longum CECT 7347 reduced the numbers of the Bacteroides fragilis group $(P=0.020)$ and the content of sIgA in stools $(P=0.011)$ compared with the administration of placebo. Although this is a first exploratory intervention with limitations, the findings suggest that $B$. longum CECT 7347 could help improve the health status of CD patients who tend to show alterations in gut microbiota composition and a biased immune response even on a GFD.
\end{abstract}

Key words: Coeliac disease: Bifidobacterium longum CECT 7347: Probiotics: Gluten-free diet

Coeliac disease (CD) is an autoimmune enteropathy caused by a permanent intolerance to dietary proteins of wheat, barley and rye in genetically predisposed (human leucocyte antigen (HLA)-DQ2/8) individuals. In CD patients, gluten proteins induce a deregulated immune response that typically leads to severe small-intestinal mucosal injury, malabsorption and gastrointestinal symptoms ${ }^{(1)}$. Gluten proteins are the main environmental factor involved in CD pathogenesis; however, recent studies have shown that other factors influencing the interplay between the gut microbiota and the mucosal immune system, including breast-feeding and gastrointestinal infections, could contribute to determining the disease onset ${ }^{(2)}$. Observational studies conducted so far have reported associations between intestinal dysbiosis and $\mathrm{CD}^{(3)}$, with a few exceptions ${ }^{(4,5)}$. Our studies have reported that the duodenal and faecal microbiota of CD patients is unbalanced with decreased numbers of Bifidobacterium spp. and increased numbers of Bacteroides spp. and Escherichia coli clones with higher virulence features, which are only partially normalised after a long-term gluten-free diet (GFD) ${ }^{(6-8)}$. One of the most recent studies has also reported that $\mathrm{CD}$ patients with gastrointestinal symptoms had different microbiota composition in comparison with controls and patients with dermatitis herpetiformis, suggesting that the microbiota may play a role in the manifestation of the disease ${ }^{(9)}$. In vitro and animal studies have demonstrated that the dysbiotic gut microbiota of CD patients and specific isolates may potentially enhance the inflammatory response elicited by gluten and

Abbreviations: CD, coeliac disease; GFD, gluten-free diet; HLA, human leucocyte antigen; sIgA, secretory IgA; tTG, tissue transglutaminase. 
contribute to increasing intestinal epithelial permeability and gliadin peptide translocation ${ }^{(10-13)}$. In contrast, Bifidobacterium longum CECT 7347 has been shown to attenuate the inflammatory effects of the dysbiotic gut microbiota of CD patients on peripheral blood mononuclear cells partially via the induction of IL-10 production ${ }^{(10,14)}$. This strain has also been proven to reduce the cytotoxic and inflammatory effects of gliadin peptides on epithelial cells in vitro via the degradation of gliadin peptides ${ }^{(15,16)}$. The administration of B. longum CECT 7347 has also been shown to reduce the numbers of peripheral $\mathrm{CD}^{+} \mathrm{T}$ cells, and increase IL-10 and reduce TNF- $\alpha$ production in jejunal sections in an animal model of gliadininduced enteropathy ${ }^{(17)}$.

The only current treatment for CD is adherence to a strict GFD, which usually results in symptomatic, serological and histological remission ${ }^{(18,19)}$. However, compliance with a strict GFD is very difficult due to the presence of gluten in most processed foods and the social restriction implied; thus, overall, the GFD is far from optimal. In practice, CD patients may continue suffering from clinical symptoms, nutritional deficiencies and higher health risks ${ }^{(20-22)}$. For example, it has been reported that even under a GFD, CD children ${ }^{(23)}$ and women ${ }^{(24)}$ have a much higher prevalence of gastrointestinal symptoms than do controls, and they also use healthcare services more often ${ }^{(22)}$. Whether or not this is due to residual chronic inflammation, exposure to minimum amounts of gluten and/or intestinal dysbiosis remains unknown. Whether complete normalisation of lymphocyte subsets in peripheral blood can be achieved remains unclear ${ }^{(19,25)}$. Other studies have also indicated that small-intestinal bacterial overgrowth is often associated with non-responsive $\mathrm{CD}^{(26)}$. The duodenal and faecal microbiota of $\mathrm{CD}$ patients has also been shown not to be completely normalised after adherence to a GFD, which can also contribute to the lack of complete restoration of intestinal immune homeostasis in those patients ${ }^{(6,7)}$. Therefore, adjunctive strategies that can improve the effects of a GFD are being investigated ${ }^{(27)}$.

The aim of the present study was to conduct a 3-month double-blind, randomised, placebo-controlled intervention trial in children with newly diagnosed CD following a GFD in order to evaluate the effects of B. longum CECT 7347 administration on immune and anthropometric parameters, and on intestinal microbiota composition.

\section{Materials and methods}

\section{Subjects and study design}

A total of thirty-six children were recruited at the Hospital Universitari Sant Joan (Reus, Tarragona) and Hospital Universitario Sant Joan de Deu (Barcelona) from November 2011 to June 2012. The inclusion criteria were children aged 2-17 years with newly diagnosed CD according to the European Society of Pedriatric Gastroenterology Hepatology and Nutrition (ESPGHAN) criteria, based on symptoms, positive antitissue transglutaminase (tTG) antibodies quantified using a tTG-IgA ELISA kit (Pharmacia Diagnostics GmbH) and duodenal biopsy demonstrating villous atrophy. The exclusion criteria did not allow subjects enrolled in another clinic study, treated with antibiotics $30 \mathrm{~d}$ before starting the study and/or with food allergy or others pathologies. Demographic and clinical characteristics of the subjects included in the study are shown in Table 1.

A double-blind, randomised, placebo-controlled intervention trial was conducted. A graphical representation of the study protocol is shown in Fig. 1. All children who were recruited ( $n$ 36) met the inclusion criteria and were randomly assigned to either the B. longum CECT 7347 ( $n$ 18) or the placebo ( $n$ 18) group. The randomisation design was generated using the SAS module PROC PLAN (version 9.1.3, service pack 2; SAS Institute Inc.) multiple of two sized blocks and a 1:1 pattern through a centralised randomisation list. The test group received B. longum CECT 7347 $\left(10^{9}\right.$ colony-forming units/capsule per d) and the placebo group received an indistinguishable capsule containing only the excipient (skimmed milk with $30 \%$ sucrose and $0.5 \%$ vitamin $\mathrm{C}$ per d) taken at lunchtime or with other food intake, for 3 months after CD diagnosis and in parallel with the GFD. Capsule groups were labelled with codes consisting of a letter and three numbers assigned randomly by an external scientist involved in bacterial strain production but not in its evaluation in the present study. Study personnel, including clinicians and research assistants involved in experimental work and data analysis, were naive as to which treatment these labels represented. All these measures ensured true allocation concealment and proper blinding. Visits to paediatricians and parameters monitored are shown in Fig. 1. The trial was not registered because it was a first exploratory study.

\section{Ethical considerations}

The study was approved by the ethics committees of Consejo Superior de Investigaciones Científicas (CSIC) and the hospitals involved in the study (Hospital Universitario Sant Joan (Reus, Tarragona) and Hospital Universitario Sant Joan de Deu (Barcelona)), and conducted in accordance with the 1975 Declaration of Helsinki as revised in 1983. Written informed consent was obtained from the parents of the children included in the study.

\section{Gluten-free diet and dietary records}

Children adhered to a strict GFD, after confirmation of CD diagnosis, by replacing gluten-containing foods by equivalent ones certified as gluten free $(<20$ parts per million $(\mathrm{mg} / \mathrm{kg})$ according to the EC Regulation no. 41/2009). Dietitians provided dietary counselling to select naturally and certified gluten-free products. They were also advised not to consume prebiotic- and probiotic-containing foods. To control diet as a variable, food diary records were collected for $72 \mathrm{~h}$ (two weekdays and one weekend day) before the start of the intervention and at the end of the 3-month intervention. At the front of the diary, detailed information on how to record food and beverages consumed using common household measures was provided. Food diary records were returned to the dietitian for their analysis for energy, macronutrient

\footnotetext{
cenal biopsy demonstrating villous atrophy. The exclusion
} 
Table 1. Clinical characteristics of the subjects of the two study groups

(Mean values with their standard errors; medians and interquartile ranges (IQR))

\begin{tabular}{|c|c|c|c|c|c|}
\hline \multirow[b]{3}{*}{ Characteristics } & \multicolumn{4}{|c|}{ Study group } & \multirow[b]{3}{*}{$P^{*}$} \\
\hline & \multicolumn{2}{|c|}{ Placebo } & \multicolumn{2}{|c|}{$\begin{array}{l}\text { Bifidobacterium } \\
\text { longum CECT } 7347\end{array}$} & \\
\hline & Mean & SE & Mean & SE & \\
\hline Cases $(n)$ & \multicolumn{2}{|c|}{16} & \multicolumn{2}{|c|}{17} & \\
\hline $\operatorname{Sex}(n)$ & & & \multirow{2}{*}{\multicolumn{2}{|c|}{7}} & 0.437 \\
\hline Male & \multicolumn{2}{|c|}{8} & & & \\
\hline Female & \multicolumn{2}{|c|}{8} & \multicolumn{2}{|c|}{10} & \\
\hline Age (years) & 8.5 & 1.2 & $6 \cdot 8$ & 0.9 & 0.265 \\
\hline Baseline height $(\mathrm{cm})$ & 26.5 & 3.8 & $21 \cdot 8$ & 3.4 & 0.301 \\
\hline Baseline weight $(\mathrm{kg})$ & $119 \cdot 8$ & $5 \cdot 7$ & $109 \cdot 3$ & $6 \cdot 4$ & 0.449 \\
\hline Height percentile (baseline/post-intervention) & & & & & $0.530 / 0.530$ \\
\hline Children above percentile 50 & \multicolumn{2}{|c|}{$2 / 2$} & \multicolumn{2}{|c|}{$3 / 3$} & \\
\hline Children at percentile 50 & \multicolumn{2}{|c|}{$0 / 0$} & \multicolumn{2}{|c|}{$0 / 0$} & \\
\hline Children under percentile 50 & \multicolumn{2}{|c|}{$14 / 14$} & \multicolumn{2}{|c|}{$14 / 14$} & \\
\hline Increased height percentile & $7 \cdot 0$ & 1.5 & $7 \cdot 8$ & $2 \cdot 2$ & 0.591 \\
\hline Post-intervention-baseline/baseline & & & & & $0.048^{\star}$ \\
\hline Median & \multicolumn{2}{|c|}{0.30} & \multicolumn{2}{|c|}{1.29} & \\
\hline IQR & \multicolumn{2}{|c|}{$0.1-0.53$} & \multicolumn{2}{|c|}{$0.22-2.85$} & \\
\hline Weight percentile (baseline/post-intervention) & & & & & $0.530 / 0.365$ \\
\hline Children above percentile 50 & \multicolumn{2}{|c|}{$3 / 3$} & \multicolumn{2}{|c|}{$4 / 6$} & \\
\hline Children at percentile 50 & \multicolumn{2}{|c|}{$0 / 1$} & \multicolumn{2}{|c|}{$0 / 0$} & \\
\hline Children under percentile 50 & \multicolumn{2}{|c|}{$13 / 12$} & \multicolumn{2}{|c|}{$13 / 11$} & \\
\hline Increased weight percentile & 3.2 & $1 \cdot 7$ & $5 \cdot 7$ & 1.8 & 0.186 \\
\hline Post-intervention - baseline/baseline & & & & & 0.234 \\
\hline Median & \multicolumn{2}{|c|}{0.22} & \multicolumn{2}{|c|}{0.26} & \\
\hline IQR & 0.0 & & 0.1 & & \\
\hline Histology (Marsh categorisation)† & & & & & 0.701 \\
\hline Marsh 1 & & & & & \\
\hline Marsh 2 & & & & & \\
\hline Marsh 3a & & & & & \\
\hline Marsh $3 b$ & & & & & \\
\hline Marsh 3c & & & & & \\
\hline $\begin{array}{l}\text { Anti-transglutaminase antibodies } \\
\text { (post-intervention - baseline/baseline) }\end{array}$ & & & & & 0.418 \\
\hline Median & & & & & \\
\hline IQR & $-0.8 \varepsilon$ & & $-0.8 \varepsilon$ & .75 & \\
\hline
\end{tabular}

and micronutrient contents based on the CESNID food composition database of Spanish foods ${ }^{(28)}$.

\section{Clinical assessments}

At baseline and after the 3-month intervention period, serological markers of $\mathrm{CD}$, anthropometric parameters (height and weight) and evolution of clinical symptoms were assessed by paediatricians specialised in gastroenterology. Parents were also instructed to register the data on compliance with probiotic intake, gastrointestinal symptoms (presence or absence of vomiting, diarrhoea, constipation or abdominal pain) and concomitant treatments (e.g. antibiotics, drugs interfering with intestinal function, etc.), and serious adverse events (hospitalisation, any life-threatening event, etc.) on a weekly basis and to report them to the paediatrician at each visit. Compliance was considered to be accomplished when the intake of the test capsules was not interrupted for more than two consecutive days and no more than three times during the whole intervention period ( 3 months). Parents were also provided a round-the-clock call service and e-mail address for immediate reporting if necessary.

\section{Lymphocyte phenotyping}

At baseline and at the end of the 3-month intervention period, peripheral blood samples were collected in heparin tubes and separated into two aliquots; one was mixed with stabilising blood Transfix reagent (Cytomark) and sent immediately to IATA-CSIC at room temperature for cytometry analysis. The second blood sample was centrifuged $\left(1200 \mathrm{~g}, 10 \mathrm{~min}, 4^{\circ} \mathrm{C}\right)$ and serum was stored at $-80^{\circ} \mathrm{C}$. Furthermore, a routine haemogram was carried out with all the blood samples. Flow cytometry was used to determine the expression of surface markers of different lymphocyte subsets. Aliquots $(100 \mu \mathrm{l})$ of stabilised peripheral blood were incubated with different 


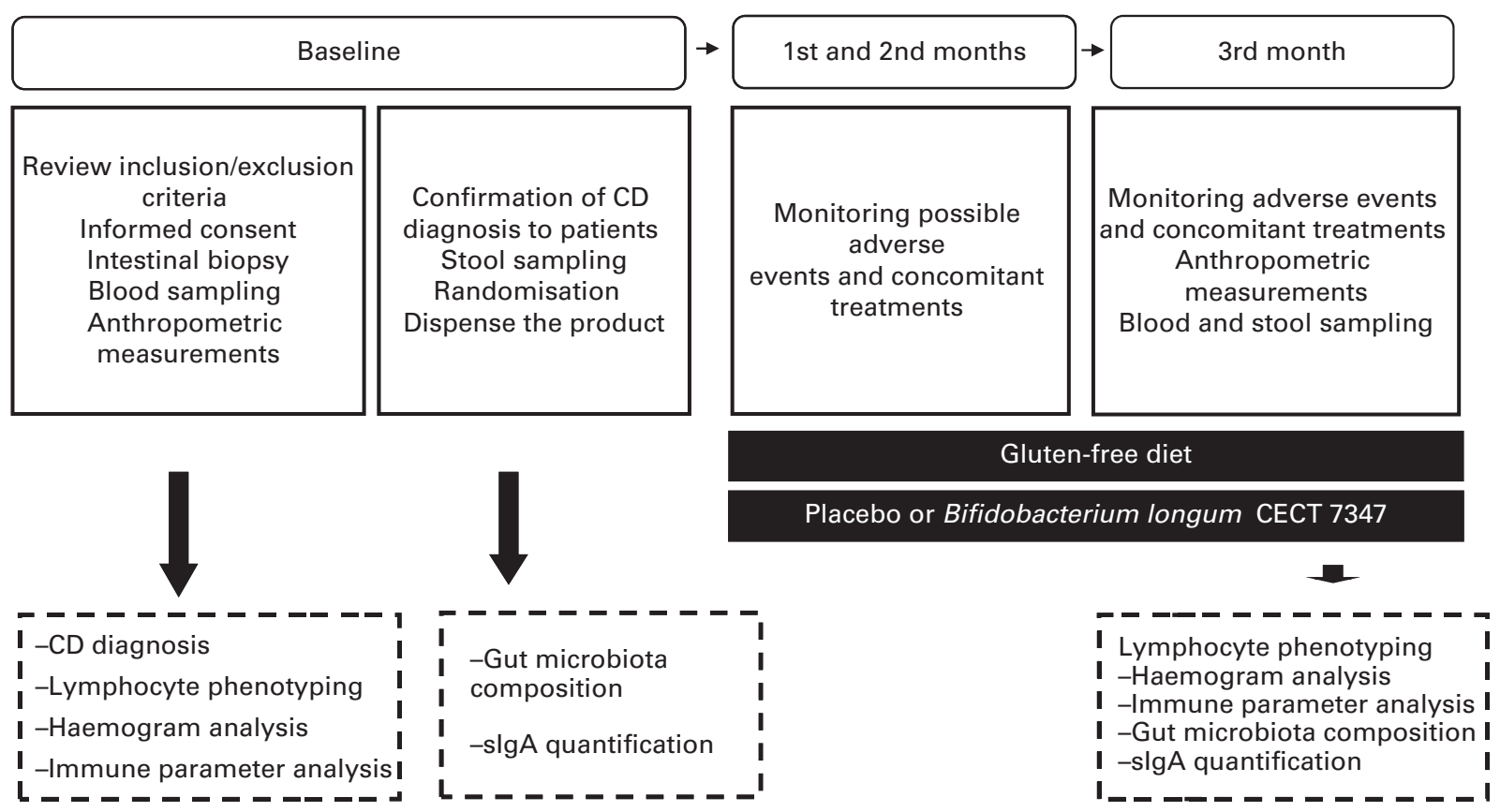

Fig. 1. Graphical representation of the study protocol and the timing for sample collection and assessments during the 3 months of intervention. $C D$, coeliac disease; slgA, secretory IgA.

fluorochrome-conjugated antibodies (eBiosciences) at room temperature for $10 \mathrm{~min}$ and then prepared for flow cytometry analysis using the Immunoprep kit (Beckman Coulter), according to the manufacturer's instructions. The samples were analysed in a FACS-Canto II Deckton Dickinson cytometer (BD Bioscience). To analyse the subsets of T-lymphocytes, cells were stained with the following monoclonal antibodies: CD45phycoerythrin (clone HI30); CD3-allophycocyanin (clone OKT3); HLA-DR-fluorescein isothiocyanate (clone LN3); CD4-fluorescein isothiocyanate (clone OKT4); Foxp3-allophycocyanin (clone PCH101); CD8-allophycocyanin (clone RPA-T8). Lymphocyte subsets were classified based on the pattern of their surface marker expression as follows: $\mathrm{CD} 45^{+} \mathrm{CD} 3^{+}$ (mature T cells); CD $45^{+} \mathrm{HLA}^{-\mathrm{DR}^{+}}$(late activation marker positive $\mathrm{T}$ cells); $\mathrm{CD} 45^{+} \mathrm{CD} 4^{+}$(helper $\mathrm{T}$ cells); $\mathrm{CD} 45^{+} \mathrm{CD} 4^{+}$and Foxp $3^{+}$(regulatory $\mathrm{T}$ cells); $\mathrm{CD} 45^{+} \mathrm{CD}^{+}$(cytotoxic $\mathrm{T}$ cells). Data from 100000 events were acquired, and percentages of each subpopulation related to the gated lymphocytes labelled with the leucocyte marker $\mathrm{CD} 45^{+}$were estimated using the BD FACSDiva ${ }^{\mathrm{TM}}$ software version 5.0 (BD Bioscience).

\section{Cytokine and Ig quantification}

In serum samples, the initial and final contents of the pro- and anti-inflammatory cytokines (TNF- $\alpha$, interferon- $\gamma$, IL-13 and IL-10) were quantified using a commercial ELISA kit following the manufacturer's instructions (catalogue no. 31673019, 31333539 and 31330139; Immunotools; catalogue no. 430605; Biolegend). Analysis of transforming growth factor- $\beta 1$ included the activation of the molecule (incubation with $1 \mathrm{M}-\mathrm{HCl}$ for $1 \mathrm{~h}$ and neutralisation with $0.5 \mathrm{M}-\mathrm{NaOH}$ ) before its quantification with an ELISA (catalogue no. 437708; Biolegend). IgG1 (catalogue no. ELH-IGG1-001; RayBiotech) and IgG4 (catalogue no. CSB-E13728h; Cusabio) contents were also quantified using specific ELISA kits.

\section{Secretory IgA quantification}

Faecal samples were collected at baseline and at the end of the intervention trial and stored at $-80^{\circ} \mathrm{C}$. Briefly, $1 \mathrm{~g}$ of the faecal sample was weighed, diluted 1:10 (w/v) with PBS buffer $(130 \mathrm{~mm}-\mathrm{NaCl}$ and $10 \mathrm{~mm}$-sodium phosphate-buffered saline, $\mathrm{pH} 7$.4) and homogenised in a stomacher for $5 \mathrm{~min}$. The mixture was also homogenised in a tube with glass beads of $5 \mathrm{~mm}$ using a vortex and subjected to low-speed centrifugation $(600 \mathrm{~g}, 2 \mathrm{~min})$. The supernatants were collected for secretory IgA ( $\operatorname{IgA}$ ) quantification by ELISA, according to the manufacturer's instructions (catalogue no. E80-102; Bethyl), as well as for DNA extraction as described below.

\section{Microbiota analysis by real-time $P C R$}

DNA was extracted using the QIAamp DNA Stool Mini Kit (Qiagen) following the manufacturer's instructions. Real-time PCR analyses were run to quantify the content of different bacterial groups and Bifidobacterium spp. using specific primers as described previously (see online supplementary Table S1). PCR amplification was performed in a LightCycler ${ }^{\circledR} 480$ RealTime PCR System (Roche) in a multi-well plate with $15 \mu \mathrm{l}$ of reaction mixture that consisted of $7.5 \mu \mathrm{l}$ SYBR Green PCR Master Mix (Roche), $3.5 \mu$ l DNase-free water, $0.75 \mu$ l of each specific primer $(10 \mathrm{~mm})$ and $2.5 \mu \mathrm{l}$ of the DNA sample. $16 \mathrm{~S}$ rRNA gene copy numbers were calculated by comparing the cycle threshold $\left(C_{\mathrm{t}}\right)$ values obtained with those from a standard curve ${ }^{(29)}$. Standard curves were generated from serial dilutions of a known copy number of the target gene 
cloned into a plasmid vector. For each reference strain, the $16 \mathrm{~S}$ rRNA gene was cloned into a pGEM-T Easy Vector System (Promega) and an E. coli $\mathrm{DH} 5 \alpha$ strain was transformed with the recombinant plasmids. Plasmid DNA was extracted from E. coli by the miniprep method ${ }^{(30)}$.

\section{Statistical analyses}

Data distribution of continuous variables was tested using the Shapiro-Wilk $W$ test. When data showed a normal distribution $(P>0.05)$, a $t$ test for comparisons of paired samples was used, and results are expressed as means with their standard errors of the mean. For non-normally distributed data, the Mann-Whitney $U$ test was used for comparisons of paired samples, and data are expressed as medians and interquartile ranges. Microbiological data were transformed from exponential numbers into logarithms to adjust to normal. All parameters measured during the intervention period are expressed as the difference between the baseline value and the post-intervention value, divided by the baseline value for pair comparisons between the two study groups using either the $t$ test or the Mann-Whitney $U$ test. A generalised linear model was also applied to absolute values of all parameters measured to establish the differences between the two study groups and possible time $\times$ treatment interactions. Correlations between the microbiota composition data and immunological data were established by using the Pearson coefficient.

Categorical demographic data of the study subjects (Table 1) and the incidence of gastrointestinal symptoms (Table 2) are expressed as absolute values or proportions, and analysed using the Fisher test.

Statistical analyses were conducted using the SPSS software (version 19.0; SPSS, Inc.) and in all the cases, statistically significant differences were considered at a $P$ value $<0.05$.

\section{Results}

\section{Characteristics of the trial subjects}

From November 2011 to June 2012 a group of thirty-six children newly diagnosed with $\mathrm{CD}$ were enrolled in a double-blind, randomised, placebo-controlled intervention

Table 2. Number of cases self-reporting gastrointestinal symptoms at least once during the 3 months of intervention together with the gluten-free diet

\begin{tabular}{|c|c|c|c|c|c|c|}
\hline & \multicolumn{2}{|c|}{$\begin{array}{c}\text { Placebo } \\
\text { group }(n 16)\end{array}$} & \multirow[b]{2}{*}{$P^{*}$} & \multicolumn{2}{|c|}{$\begin{array}{l}\text { Bifidobacterium } \\
\text { longum CECT } \\
7347 \text { group }(n 17)\end{array}$} & \multirow[b]{2}{*}{$P^{\star}$} \\
\hline & Initial & Final & & Initial & Final & \\
\hline Diarrhoea & 6 & 4 & 0.352 & 6 & 5 & 0.500 \\
\hline Constipation & 3 & 2 & 0.500 & 3 & 2 & 0.500 \\
\hline Abdominal pain & 6 & 4 & 0.352 & 10 & 9 & 0.500 \\
\hline Vomiting & 5 & 1 & 0.087 & 4 & 1 & 0.206 \\
\hline
\end{tabular}

* Data were compared using the Fisher test and significant differences were established at a $P$ value $<0.05$. trial to evaluate the effects of the intervention with B. longum CECT 7347 together with those of the GFD on the gut ecosystem. There were three withdrawals registered: one due to the parents' complaints of the child's reduced appetite and two due to the intake of antibiotics. Of these withdrawals, two belonged to the placebo group and one to the B. longum group. Finally, data from seventeen subjects of the intervention group and sixteen of the placebo group were included in the statistical analyses. Taking into account the number of the children enrolled, the mean missed doses were 1.3 and 1.9 in the placebo and intervention groups, respectively, which did not differ significantly $(P=0 \cdot 193)$.

Dietary records did not reveal significant differences between the groups in terms of the intake of energy and specific nutrients $(P>0.05)$. The adherence to the GFD only led to a slight reduction in the intake of dietary fibre in the children of both groups (see online supplementary Table S2). Intake of other probiotics or prebiotics was not reported during the study period.

The demographic and clinical characteristics of children from the two groups are shown in Table 1. Proper randomisation was achieved as there were no significant differences in the baseline characteristics between the two groups. Serum antibodies to tTG-IgA were normalised, decreasing to similar levels $(P=0.418)$ in both groups after 3 months of adherence to a GFD and the intervention (Table 1). No adverse events were reported during the intervention. Height and weight data were analysed according to the WHO growth-chart percentiles. As is shown in Table 1 , at the time of diagnosis, $84.8 \%(n 28 / 33)$ and $78.8 \%$ ( $n$ 26/33) of the children enrolled in the study were under percentile 50 for height and weight, respectively, corresponding to their age. After 3 months of intervention, two children in the B. longum CECT 7347 group and one child in the placebo group gained weight so they were included in the group of children above percentile 50 and in the group of children at percentile 50, respectively. The mean increases in height and weight percentiles (difference in the percentile from baseline and post-intervention) were higher in the B. longum CECT 7347 group $(\Delta 7 \cdot 8$ (SE 2.2) and $\Delta 5 \cdot 7$ (SE 1.8 ), respectively) than in the placebo group $(\Delta 7 \cdot 0$ (SE 1.5$)$ and $\Delta 3 \cdot 2$ (SE $1 \cdot 7)$, respectively). Comparison of these changes between the groups revealed that increases in height percentile were significantly greater in the B. longum CECT 7347 group than in the placebo group $(P=0 \cdot 048)$, while statistically significant differences in weight percentile increases were not detected $(P=0 \cdot 234)$.

The incidence of gastrointestinal symptoms (diarrhoea, constipation, abdominal pain and vomiting) in the two study groups during the 3 months of intervention is shown in Table 2. No statistically significant differences in the number of cases self-reporting the incidence of these symptoms were detected either between the groups or within the placebo or B. longum group.

\section{Lymphocyte phenotype and haemogram}

The results of immune phenotyping of peripheral blood cells by flow cytometry are shown in Table 3 and Fig. 2. 
Table 3. Changes in the subsets of $C D 45^{+} \mathrm{T}$ lymphocytes within the placebo or Bifidobacterium longum CECT 7347 group during the 3 months of intervention together with the gluten-free diet

(Mean values with their standard errors)

\begin{tabular}{|c|c|c|c|c|c|}
\hline & \multicolumn{2}{|c|}{ Baseline } & \multicolumn{2}{|c|}{$\begin{array}{c}\text { Post- } \\
\text { intervention }\end{array}$} & \multirow[b]{2}{*}{$P^{*}$} \\
\hline & Mean & SE & Mean & SE & \\
\hline \multicolumn{6}{|l|}{ Placebo group } \\
\hline $\mathrm{CD}^{+}$ & 66.96 & $2 \cdot 85$ & $70 \cdot 03$ & $2 \cdot 17$ & 0.144 \\
\hline $\mathrm{HLA}^{-\mathrm{DR}^{+}}$ & 0.99 & 0.19 & 0.72 & 0.24 & 0.422 \\
\hline $\mathrm{CD}^{+}$ & 41.78 & $4 \cdot 37$ & $42 \cdot 60$ & 4.89 & 0.898 \\
\hline $\mathrm{CD}^{+}{ }^{\mathrm{Foxp}} 3^{+}$ & 2.99 & 0.82 & 3.77 & 1.36 & 0.322 \\
\hline $\mathrm{CD}^{+}$ & 22.08 & 1.95 & $20 \cdot 68$ & $1 \cdot 13$ & 0.344 \\
\hline \multicolumn{6}{|c|}{ B. longum CECT 7347 group } \\
\hline $\mathrm{CD}^{+}$ & $70 \cdot 05$ & $2 \cdot 23$ & 63.42 & 2.04 & 0.013 \\
\hline $\mathrm{HLA}^{-\mathrm{DR}^{+}}$ & 0.62 & $0 \cdot 10$ & 0.38 & 0.82 & 0.029 \\
\hline $\mathrm{CD}^{+}$ & 44.68 & $2 \cdot 40$ & $45 \cdot 96$ & 3.59 & 0.748 \\
\hline $\mathrm{CD}^{+} \mathrm{Foxp}^{+}$ & $3 \cdot 13$ & 0.66 & 3.45 & 0.73 & 0.728 \\
\hline $\mathrm{CD}^{+}$ & 23.92 & 1.55 & 21.59 & 0.98 & 0.152 \\
\hline
\end{tabular}

* Differences between the baseline and post-intervention values within the study groups (placebo or $B$. longum CECT 7347) were established at $P<0.05$ using the two-sided $t$ test.

Within-group comparisons did not reveal statistically significant changes in the placebo group during the 3 months of intervention when comparing the baseline values with the post-intervention ones (Table 3). In contrast, B. longum CECT 7347 administration led to significant decreases in $\mathrm{CD}^{+}(P=0.013)$ and HLA-DR ${ }^{+}(P=0.029)$ T-cell populations when comparing the baseline values with the postintervention ones within this group (Table 3). Baseline values of the measured parameters were not significantly different between the groups. Comparisons between the groups revealed that the administration of B. longum CECT 7347 significantly reduced $(P=0.004)$ the $\mathrm{CD}^{+}{ }^{+}$T-cell population when compared with placebo treatment (Fig. 2). No statistically significant differences were detected in the subsets of $\mathrm{T}$ lymphocytes HLA-DR ${ }^{+}, \mathrm{CD}^{+}, \mathrm{CD}^{+}{ }^{+} \mathrm{Foxp}^{+}$and $\mathrm{CD} 8^{+}$ between the groups $(P=0.328, P=0.970, P=0.504$ and $P=0.376$, respectively; Fig. 2 ).

Similar differences were detected between the groups by applying a generalised linear model. This model also revealed a significant effect of the intervention with B. longum CECT 7347, resulting in a reduction of the $\mathrm{CD}^{+}$population that was opposite to the effect of the treatment with the GFD $(P=0.020)$ and a statistically significant time $\times$ treatment interaction $(P=0.021)$. The baseline values for $\mathrm{CD}^{+}$were not different between the two groups $(P=0.950)$.

No changes in the counts of total leucocytes and leucocyte subtypes (lymphocytes, monocytes, granulocytes, eosinophils and basophils) or $\mathrm{Hb}$ content were observed on analysing the haemogram (data not shown), either within or between the groups.

\section{Cytokines and Ig content}

The results of serum cytokines and Ig concentrations are shown in Table 4 and Fig. 3. Within-group comparisons revealed that the administration of B. longum CECT 7347 slightly reduced serum TNF- $\alpha$ concentrations, but differences did not reach statistical significance $(P=0.085)$. Changes in other serum cytokines and Ig concentrations, comparing the baseline and post-intervention values within the groups, were not significantly different in the B. longum CECT 7347 or the placebo group (Table 4). Comparisons between the groups (B. longum CECT 7347 and placebo) for changes in serum cytokines and Ig levels are shown in Fig. 3. The baseline values of the measured parameters were not significantly different between the groups. The administration of B. longum CECT 7347 slightly reduced serum TNF- $\alpha$ concentration compared with the placebo treatment, but did not reach statistical significance $(P=0.067)$. No statistically significant differences were detected in the concentrations of serum IL-10, interferon- $\gamma$, IL-13, transforming growth factor- $\beta 1, \operatorname{IgG} 1$ and IgG 4 between the groups.

In the generalised linear model, significant differences in IgG4 ( $P=0.038)$ were detected, showing increased values for the placebo group. Significant effects or interactions were not detected for other parameters.

\section{Microbiota composition and faecal secretory IgA content}

Within-group changes in microbiota composition as a result of the intervention showed that the GFD plus placebo administration led to a statistically significant increase in gene copy numbers of the Bacteroides fragilis group $(P=0.013)$ and in the family Enterobacteriaceae $(P=0.038)$ (placebo group; Table 5). The gene copy numbers of Bifidobacterium spp. were also slightly reduced as a consequence of the GFD, although differences did not reach statistical significance $(P=0 \cdot 151)$. Within-group comparisons also showed that the ratio of Lactobacillus group + Bifidobacterium spp./B. fragilis group + Enterobacteriaceae was significantly reduced in the placebo group (1.26 (SE 0.08) v. 1.00 (SE 0.04); $P=0.006$ ), while the $B$. longum CECT 7347 group did not show statistically significant differences $\left(\begin{array}{lllll}1 \cdot 19 & \text { (SE } & 0 \cdot 07\end{array}\right)$ v. 1.16

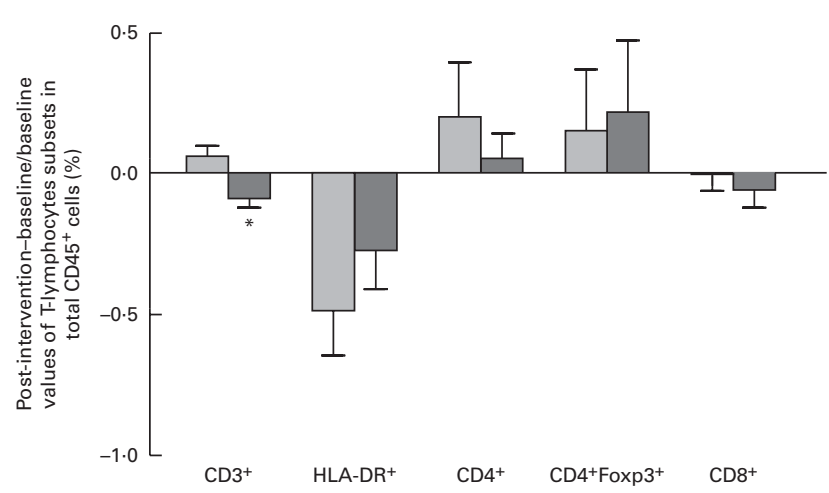

Fig. 2. Changes in the subsets of $C D 45^{+}$estimated as the difference between the baseline value and the post-intervention value, divided by the baseline value of the placebo ( $\square$ ) and Bifidobacterium longum CECT 7347 ( $\square$ ) groups during the 3 months of intervention together with the gluten-free diet. Values are means, with their standard errors represented by vertical bars. * Mean value was significantly different from that of the placebo group $(P<0.05$; two-sided Mann-Whitney $U$ test). HLA, human leucocyte antigen. 
Table 4. Changes in the contents of cytokines $(\mathrm{pg} / \mathrm{ml})$ and $\mathrm{lg}(\mathrm{ng} / \mathrm{ml})$ within the placebo or Bifidobacterium longum CECT $7347 \mathrm{group}$ during the 3 months of intervention together with the gluten-free diet

(Mean values with their standard errors)

\begin{tabular}{|c|c|c|c|c|c|}
\hline & \multicolumn{2}{|c|}{ Baseline } & \multicolumn{2}{|c|}{ Post-intervention } & \multirow[b]{2}{*}{$P^{*}$} \\
\hline & Mean & SE & Mean & SE & \\
\hline \multicolumn{6}{|c|}{ Placebo group } \\
\hline TNF- $\alpha$ & $1669 \cdot 56$ & 338.47 & $1515 \cdot 24$ & $254 \cdot 34$ & 0.443 \\
\hline $\mathrm{IFN}-\gamma$ & 306.42 & 32.59 & 297.59 & $36 \cdot 32$ & 0.815 \\
\hline IL-10 & 42.99 & 9.25 & $59 \cdot 30$ & 9.33 & 0.249 \\
\hline IL-13 & $137 \cdot 71$ & 12.85 & 163.86 & $21 \cdot 16$ & 0.239 \\
\hline TGF- $\beta 1$ & 116.43 & 38.62 & $146 \cdot 63$ & $25 \cdot 71$ & 0.537 \\
\hline $\lg G 1$ & 441.85 & 84.04 & 477.39 & 94.43 & 0.644 \\
\hline $\lg G 4$ & 4.63 & 0.42 & $6 \cdot 29$ & $1 \cdot 18$ & 0.212 \\
\hline \multicolumn{6}{|c|}{ B. longum CECT 7347 group } \\
\hline TNF- $\alpha$ & 1803.29 & 187.95 & 1442.94 & $153 \cdot 20$ & 0.085 \\
\hline IFN- $\gamma$ & 267.02 & 33.52 & 235.49 & 23.97 & 0.423 \\
\hline $\mathrm{IL}-10$ & $69 \cdot 14$ & $12 \cdot 15$ & 52.02 & $5 \cdot 28$ & 0.240 \\
\hline $\mathrm{IL}-13$ & $116 \cdot 21$ & $20 \cdot 85$ & 119.96 & $7 \cdot 11$ & 0.854 \\
\hline TGF- $\beta 1$ & $130 \cdot 67$ & 34.41 & 138.42 & 33.52 & 0.851 \\
\hline $\lg \mathrm{I}_{1}$ & 368.91 & 57.51 & $400 \cdot 82$ & 74.43 & 0.590 \\
\hline $\lg G 4$ & $4 \cdot 38$ & 0.19 & 4.42 & 0.34 & 0.919 \\
\hline
\end{tabular}

IFN- $\gamma$, interferon- $\gamma$; TGF- $\beta 1$, transforming growth factor- $\beta 1$.

${ }^{*}$ Differences between the baseline and post-intervention values within the placebo or $B$. longum CECT 7347 group were established at $P<0.05$ using the two-sided $t$ test.

(SE 0.07); $P=0.811$ ). Comparisons between the groups revealed that gene copy numbers of the $B$. fragilis group increased significantly in the placebo group compared with the B. longum CECT 7347 group $(P=0 \cdot 020$; Fig. 4). The quantification of SIgA content in the faeces showed that the treatments caused opposite changes within the groups, which were statistically significant for the placebo group, showing an increase in faecal sIgA concentrations $(P=0.009$; Table 5). Comparisons between the groups revealed that the administration of B. longum CECT 7347 led to significant reductions in faecal sIgA concentration compared with the placebo treatment $(P=0 \cdot 011$; Fig. 5).

In the B. longum CECT 7347 group, decreases in stool sIgA levels correlated with decreases in total bacteria and the gene copy numbers of the $B$. fragilis group $(r 0.729, P=0.005$ and $r$ 0.522, $P=0.047$, respectively; Fig. 6). No correlations were found between bacteria and other immune parameters in either the placebo or the B. longum group.

In the generalised linear model of analysis, the placebo group showed increases in the numbers of the $B$. fragilis group with borderline significance compared with the intervention group $(P=0.055)$. Significant and opposite changes in the SIgA content depending on the treatment were also detected $(P=0.031)$ as well as a time $\times$ treatment interaction $(P=0.016)$ : faecal sIgA content decreased in the B. longum CECT 7347 group, while it increased in the placebo group. The baseline values for the numbers of the $B$. fragilis group and faecal sIgA content were not different between the two groups $(P=0.972$ and $P=0.416$, respectively).

\section{Discussion}

The influence of administering B. longum CECT 7347 orally to children with newly diagnosed CD following a GFD was evaluated in a 3-month double-blind, randomised, placebocontrolled intervention trial. The aim of the present study was to assess whether the intervention in the gut ecosystem with B. longum CECT 7347 improved the efficacy of the GFD. Adherence to the GFD, with and without B. longum CECT 7347 supplementation, led to the positive effects on growth-related parameters in the children under study, as expected. Furthermore, B. longum CECT 7347 administration led to additional improvements, corresponding to greater increases in the height percentile compared with the placebo treatment. Adherence to a GFD is associated with restoring growth, measured as the increase in height percentile and the decrease in weight deficit, as reported in a cohort of

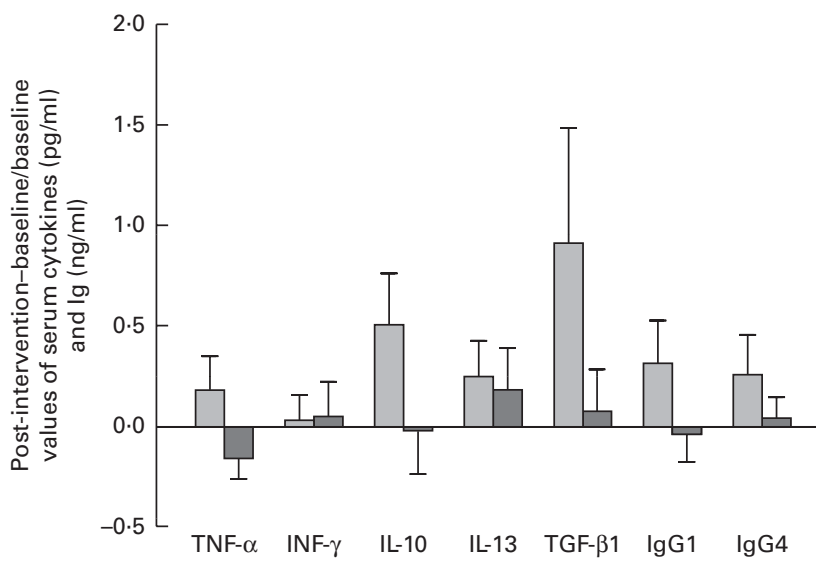

Fig. 3. Changes in the contents of cytokines and Ig estimated as the difference between the baseline value and the post-intervention value, divided by the baseline value of the placebo ( $\square$ ) and Bifidobacterium longum CECT 7347 ( $\square$ ) groups during the 3 months of intervention together with the glutenfree diet. Values are means, with their standard errors represented by vertical bars. INF- $\gamma$, interferon- $\gamma$, TGF- $\beta 1$, transforming growth factor- $\beta 1$. 
Table 5. Changes in the composition of intestinal microbiota (log gene copy numbers/g stools) within the placebo or Bifidobacterium longum CECT 7347 group during the 3 months of intervention together with the gluten-free diet

(Mean values with their standard errors)

\begin{tabular}{|c|c|c|c|c|c|}
\hline & \multicolumn{2}{|c|}{ Baseline } & \multicolumn{2}{|c|}{ Post-intervention } & \multirow[b]{2}{*}{$P^{*}$} \\
\hline & Mean & SE & Mean & $\mathrm{SE}$ & \\
\hline \multicolumn{6}{|l|}{ Placebo group } \\
\hline Total bacteria & $11 \cdot 10$ & 0.14 & $11 \cdot 15$ & 0.18 & 0.689 \\
\hline Bacteroides fragilis group & 9.07 & 0.17 & 9.59 & 0.22 & 0.013 \\
\hline Enterobacteriaceae & 6.03 & 0.38 & 6.93 & 0.34 & 0.038 \\
\hline Lactobacillus group & 8.55 & 0.14 & 8.45 & $0 \cdot 18$ & 0.589 \\
\hline Bifidobacterium spp. & 9.37 & 0.19 & 9.01 & 0.24 & 0.151 \\
\hline Clostridium coccoides group & 8.85 & 0.33 & $9 \cdot 31$ & 0.28 & 0.115 \\
\hline Clostridium leptum group & $9 \cdot 86$ & 0.23 & $10 \cdot 07$ & 0.30 & 0.434 \\
\hline $\operatorname{slgA}(\mathrm{mg} / \mathrm{ml})$ & $67 \cdot 07$ & 5.75 & $84 \cdot 15$ & $6 \cdot 01$ & 0.009 \\
\hline \multicolumn{6}{|l|}{ B. longum CECT 7347 group } \\
\hline Total bacteria & $11 \cdot 27$ & 0.15 & $11 \cdot 08$ & $0 \cdot 16$ & 0.393 \\
\hline Bacteroides fragilis group & 9.43 & 0.20 & $9 \cdot 29$ & $0 \cdot 16$ & 0.583 \\
\hline Enterobacteriaceae & 5.90 & 0.46 & 6.34 & 0.43 & 0.424 \\
\hline Lactobacillus group & 8.46 & 0.21 & 8.54 & 0.33 & 0.858 \\
\hline Bifidobacterium spp. & 9.08 & 0.27 & $9 \cdot 16$ & 0.42 & 0.875 \\
\hline Clostridium coccoides group & $9 \cdot 22$ & 0.23 & $9 \cdot 60$ & 0.42 & 0.469 \\
\hline Clostridium leptum group & $10 \cdot 23$ & 0.23 & $10 \cdot 01$ & $0 \cdot 16$ & 0.447 \\
\hline slgA (mg/g stools) & 77.53 & 4.89 & $60 \cdot 83$ & $7 \cdot 16$ & 0.109 \\
\hline
\end{tabular}

ninety children (aged $0 \cdot 5-7 \cdot 5$ years) with the disease ${ }^{(31)}$. The effect of a GFD on the growth of a cohort of sixty CD children (aged 2.3-10 years) in a 4-year study also showed that the weight-for-height parameter normalised by the end of the first year, while height recovery caught up steadily until the third year of adherence to the GFD, revealing a faster catch-up of weight than height ${ }^{(32)}$. The administration of B. longum CECT 7347 caused significant decreases in mature $\mathrm{T}$ lymphocytes $\left(\mathrm{CD}^{+}\right)$and HLA-DR ${ }^{+} \mathrm{T}$ lymphocytes, according to the within-group comparisons, while the placebo treatment did not. Moreover, there were reduced numbers of mature $\mathrm{T}$ lymphocytes $\left(\mathrm{CD}^{+}\right)$according to the betweengroup comparisons with respect to the placebo treatment. Altogether, these results indicate that the bifidobacterial strain exerted a greater effect on these lymphocyte subsets than the GFD alone, which could presumably contribute to better recovery from the inflammatory status associated with the active phase of the disease, which is characterised by increased $\mathrm{T}$-cell activation that drives an inflammatory response with increased pro-inflammatory cytokine production $^{(33)}$. Statistically significant correlations between the densities of mucosal $\mathrm{CD}^{+}{ }^{+}$with serum tTG-2 antibody levels have been established in untreated and treated CD patients, and the number of intraepithelial $\mathrm{CD}^{+}$cells was also associated with the villus height:crypt depth ratio ${ }^{(33)}$. Another study in newly diagnosed CD children (aged 2-5 years) has also reported increased peripheral prevalence of HLA-DR ${ }^{+}$cells, which was partially reduced after adherence to a $\mathrm{GFD}^{(19)}$. This is in agreement with our findings, pointing to an improved effect of the GFD combined with B. longum CECT 7347 intake on this lymphocyte subset. In the present study, only regulatory $\left(\mathrm{CD} 4^{+}\right.$Foxp $\left.3^{+}\right)$T cells tended to increase in the placebo group, and increased even more in the B. longum
CECT 7347 group together with helper $\left(\mathrm{CD}^{+}\right) \mathrm{T}$ cells. Although changes were not statistically significant, this could indicate an induction of the regulatory mechanisms controlling the unbalanced inflammatory $\mathrm{T}$-cell response associated with CD.

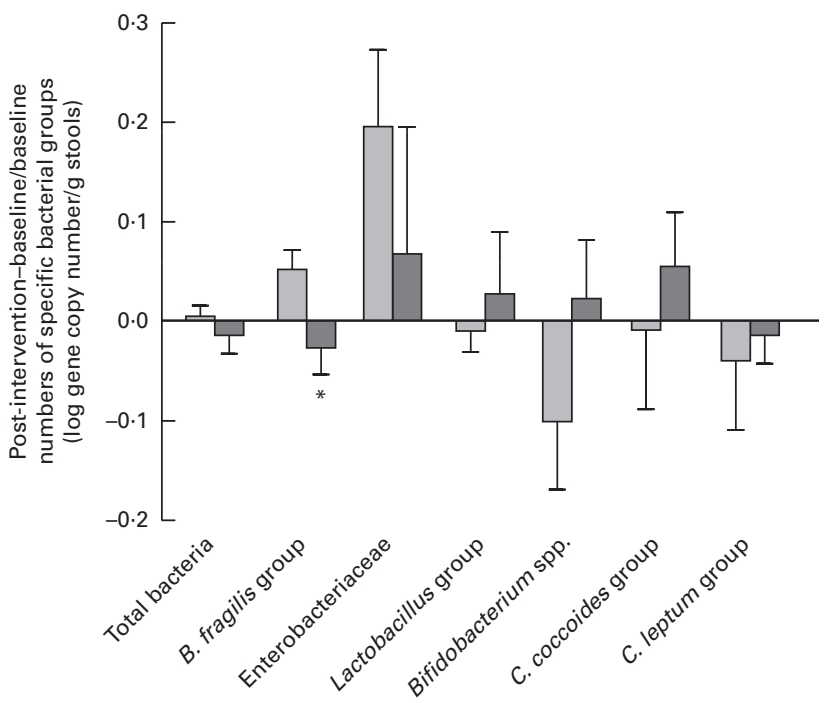

Fig. 4. Changes in the composition of intestinal microbiota (log gene copy numbers/g stools) estimated as the difference between the baseline value and the post-intervention value, divided by the baseline value of the placebo $(\square)$ and Bifidobacterium longum CECT 7347 ( $\square$ ) groups during the 3 months of intervention together with the gluten-free diet. Values are means, with their standard errors represented by vertical bars. * Mean value was significantly different from that of the placebo group $(P<0.05$; two-sided Mann-Whitney $U$ test). B. fragilis, Bacteroides fragilis; $C$. cocoides, Clostridium coccoides; C. leptum, Clostridium leptum. 


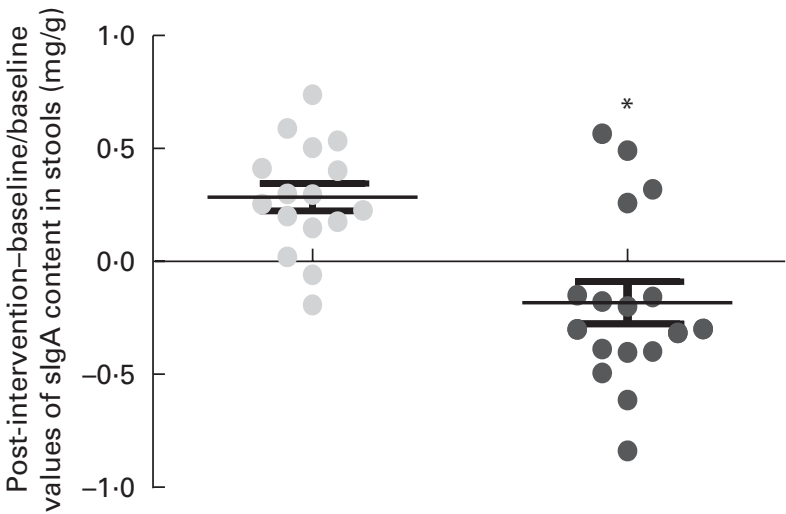

Fig. 5. Changes in the content of secretory $\lg A(\lg A)$ in stools $(\mathrm{mg} / \mathrm{g})$ estimated as the difference between the baseline value and the post-intervention value, divided by the baseline value of the placebo (O) and Bifidobacterium longum CECT 7347 (O) groups during the 3 months of intervention together with the gluten-free diet. Values are means of individual data, with their standard errors represented by vertical bars. * Mean value was significantly different from that of the placebo group $(P<0.05$; two-sided $t$ test).

Compared with the placebo treatment, B. longum CECT 7347 administration also tends to decrease serum TNF- $\alpha$ concentration, suggesting that this bifidobacterial strain could contribute to reducing the inflammatory status of patients to a greater extent than the GFD alone. This finding is also supported by a recent study in an animal model of gliadininduced enteropathy reporting that B. longum CECT 7347 administration reduced $\mathrm{TNF}-\alpha$ production in the smallintestinal tissue but increased IL-10 production ${ }^{(17)}$. Statistically significant positive correlations have been reported between IgA-anti-tTG and serum TNF- $\alpha$ in subjects with both type 1 diabetes mellitus and $\mathrm{CD}^{(34)}$. Also, patients with newly diagnosed CD have been reported to have increased TNF- $\alpha$ serum concentration ${ }^{(35)}$. In the context of CD, TNF- $\alpha$ production, together with interferon- $\gamma$, are thought to contribute to increasing intestinal epithelial permeability, favouring the access of higher antigen loads to the submucosa and, therefore, aggravating CD pathogenesis ${ }^{(36)}$. Recent studies in intestinal cultures in vitro and ex vivo have also demonstrated that TNF- $\alpha$ may contribute to exacerbating the pathogenic mechanism of CD by inducing tTG-2 expression, synergically with interferon- $\gamma^{(37)}$.

The present study also demonstrated that in children with newly diagnosed $\mathrm{CD}$, imbalances in the microbiota resulting from the GFD were counteracted by parallel administration of B. longum CECT 7347. In this respect, in the placebo group, the GFD led to significant increases in potentially pathogenic bacteria (B. fragilis group and Enterobacteriaceae) and reductions in the ratio of harmless to potentially harmful bacteria. In agreement with the present findings, increases in the ratio of the Bacteroides-Prevotella + E. coli/ Bifidobacterium + Lactobacillus group were detected in the stools of untreated and treated CD children with a GFD previously $^{(6)}$. In addition, increases in the numbers of $B$. fragilis in both stools and duodenal biopsies of patients with active and non-active diseases have been reported previously ${ }^{(7)}$. Furthermore, it was demonstrated that the abundance of $B$. fragilis species coding for metalloproteases was increased in both untreated and treated CD patients, which could presumably play a pathogenic role in $\mathrm{CD}^{(13)}$. In fact, B. fragilis and the strains producing metalloproteases are frequently involved in opportunistic infections and aggravate colitis in animal models ${ }^{(38)}$. Studies in healthy adults have also shown that the GFD per se shifts gut microbiota composition, reducing the numbers of Bifidobacterium spp., B. longum and the Lactobacillus group, and increasing those of Enterobacteriaceae and $E$. coli ${ }^{(39)}$. In vitro and animal studies have also shown that these microbiota alterations could contribute to pathogenesis in the active phase of the disease as well as to the lack of complete restoration of immune homeostasis in GFD-treated patients ${ }^{(10,12,14)}$. However, in the present study, statistically significant changes specifically in the numbers of Bifidobacterium spp. were not detected, neither associated with the adherence to the GFD nor with probiotic administration. It has been reported that changes in the microbiota resulting from a dietary intervention depend among other
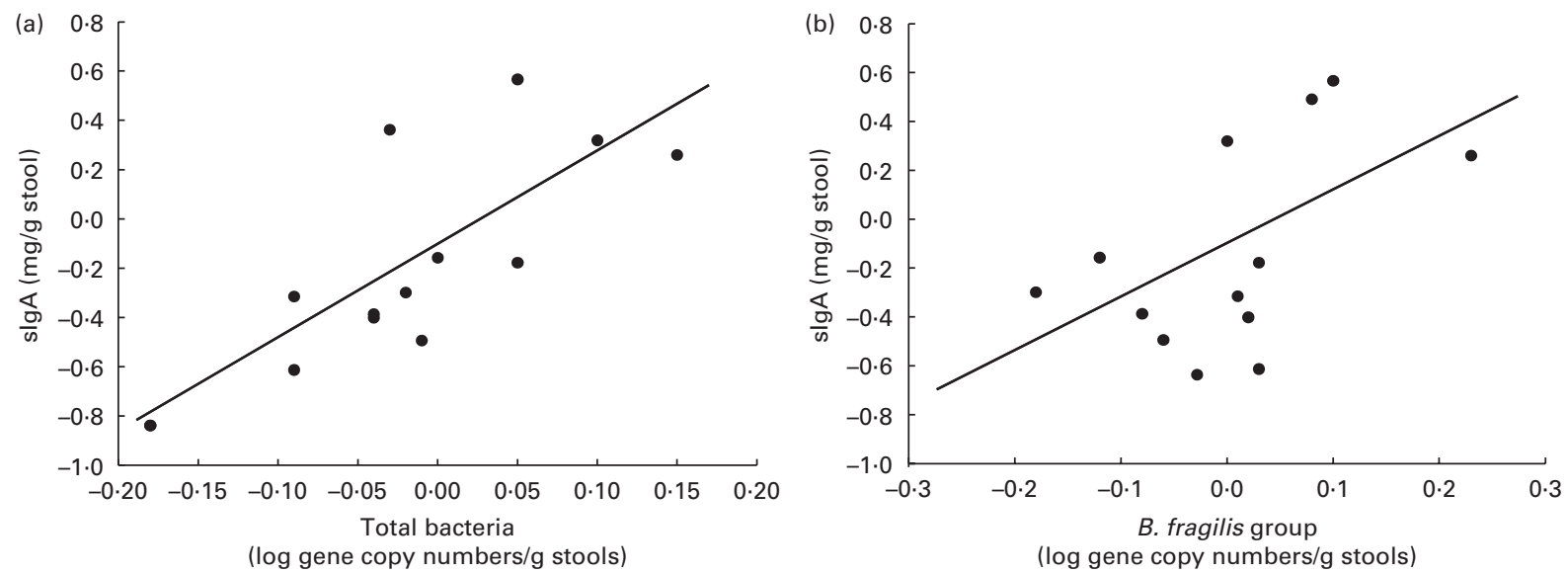

Fig. 6. Correlation analyses of the changes between the baseline and post-intervention values of secretory lgA (slgA) concentrations (mg/g stools) and log gene copy numbers of $(\mathrm{a})$ total bacteria $(r 0.729 ; P=0.005)$ and $(b)$ Bacteroides fragilis group $(r 0.522 ; P=0.047)$ in children treated with Bifidobacterium longum CECT 7347 plus the gluten-free diet. 
factors on the initial microbiota composition, and, accordingly, previous studies have indicated that changes in bifidobacterial numbers can be minor after probiotic or prebiotic administration in subjects with high initial bifidobacterial counts ${ }^{(40)}$, as it was the case for the present study. Also, according to our previous studies, the GFD does not favour the growth of bifidobateria ${ }^{(39)}$

The changes induced by the administration of B. longum CECT 7347 in the intestinal microbiota were parallel to the decreases in faecal SIgA concentration. SIgA is considered to be the most likely host secretion to affect the localisation, growth and composition of the gut microbiota, and can restrict the colonisation of harmful bacteria ${ }^{(41,42)}$. Nevertheless, it has also been speculated that gut microbiota composition may influence $\operatorname{sIgA}$ concentration and specificities as a result of a bidirectional cross-talk between the host and its microbiota $^{(41,42)}$. The fact that the administration of $B$. longum CECT 7347 led to a reduction in sIgA content that was statistically correlated with the lower gene copy numbers of total bacteria and the $B$. fragilis group would suggest a reduction in the host sIgA-mediated protective response against luminal bacterial antigens due to the restoration of the gut ecosystem with decreases in harmful bacteria ${ }^{(43)}$. A recent, double-blind, randomised, placebo-controlled study has evaluated the effect of $B$. infantis Natren Life Start (NLS) in untreated CD patients consuming gluten to establish the effect of the probiotic independently of the GFD ${ }^{(44)}$. The beneficial properties of $B$. infantis NLS included the reduction of gastrointestinal symptoms, specifically indigestion, constipation and reflux with borderline significance. However, it neither improved diarrhoea or abdominal pain, nor modified intestinal permeability or the pro-inflammatory status as reflected by the analysis of serum cytokines and chemokines ${ }^{(44)}$. Despite the experimental differences, it is presumable that the mechanisms behind the effects exerted by $B$. infantis NLS differ from those exerted by B. longum CECT 7347, as the latter influences inflammatory markers and the gut microbiota and host-related defence mechanisms.

The study reported herein has some limitations partly inherent to exploratory interventions. These include the following: small population size; lack of previous data for power calculation; inclusion of children with a wide age range, which could increase the variability of immune parameters determined; a relatively short duration to detect clinical effects. Nonetheless, the findings are interesting enough to encourage further well-powered intervention studies, which would provide sound data on the efficacy of this bifidobacterial strain and shed light on its mode of action in ameliorating inflammatory conditions such as CD.

In conclusion, the oral administration of B. longum CECT 7347 together with the GFD led to shifts in the intestinal microbiota, characterised by the reductions in potentially pro-inflammatory bacteria (B. fragilis group) related to $\mathrm{CD}$ in previous human observational studies as well as faecal sIgA. Likewise, B. longum CECT 7347 administration led to reductions in activated $\mathrm{T}$ lymphocytes and also tended to reduce inflammatory markers (TNF- $\alpha$ ) that may contribute to the recovery of immune homeostasis in $\mathrm{CD}$ patients.

\section{Supplementary material}

To view supplementary material for this article, please visit http://dx.doi.org/10.1017/S0007114514000609

\section{Acknowledgements}

The authors thank the children with CD and their parents for participating in the study, as well as Dr Karina Guadalupe Hernández and Dr Francisco Sosa for their collaboration in the sample and data collection.

The present study was supported by grants CEN-20091006 (MICINN, Spain) and Consolider Fun-C-Food CSD2007-00063 from the Spanish Ministry of Science and Innovation (MICINN, Spain). M. O. has a JAE-preDoc contract (CSIC, Spain).

The authors' contributions were as follows: Y. S. conceived the study concept and design; M. O. carried out the microbiological and immunological analysis, created the database and performed all statistical analyses; V. V. and G. C. conducted the dietary intervention and collected the clinical and dietary data; M. O. and Y. S. drafted the manuscript. All authors read and approved the final version of the manuscript.

There are no conflicts of interest.

\section{References}

1. Di Sabatino A \& Corazza GR (2009) Coeliac disease. Lancet 373, 1480-1493.

2. Pozo-Rubio T, Olivares M, Nova E, et al. (2012) Immune development and intestinal microbiota in celiac disease. Clin Dev Immunol 2012, 654143.

3. Laparra M, Olivares M \& Sanz Y (2013) Role of gut microbes in celiac disease risk and pathogenesis. In Celiac Disease. Chapter 7 [S Devi Rampertab and E Gerand, editors]. Dordrecht: Springer.

4. Kalliomäki M, Satokari R, Lähteenoja H, et al. (2012) Expression of microbiota, Toll-like receptors, and their regulators in the small intestinal mucosa in celiac disease. J Pediatr Gastroenterol Nutr 54, 727-732.

5. de Meij TG, Budding AE, Grasman ME, et al. (2013) Composition and diversity of the duodenal mucosa-associated microbiome in children with untreated coeliac disease. Scand J Gastroenterol 48, 530-536.

6. Nadal I, Donat E, Ribes-Koninckx C, et al. (2007) Imbalance in the composition of the duodenal microbiota of children with coeliac disease. J Med Microbiol 56, 1669-1674.

7. Collado MC, Donat E, Ribes-Koninckx C, et al. (2008) Imbalances in faecal and duodenal Bifidobacterium species composition in active and non-active coeliac disease. $B M C$ Microbiol 8, 232.

8. Sanz Y, De Palma G \& Laparra M (2011) Unraveling the ties between celiac disease and intestinal microbiota. Int Rev Immunol 30, 207-218.

9. Wacklin P, Kaukinen K, Tuovinen E, et al. (2013) The duodenal microbiota composition of adult celiac disease patients is associated with the clinical manifestation of the disease. Inflamm Bowel Dis 19, 934-941.

10. Medina M, De Palma G, Ribes-Koninckx C, et al. (2008) Bifidobacterium straits suppress in vitro the pro-inflammatory milieu triggered by the large intestinal microbiota of coeliac patients. I Inflamm (London) 5, 19. 
11. De Palma G, Nadal I, Medina M, et al. (2010) Intestinal dysbiosis and reduced immunoglobulin-coated bacteria associated with coeliac disease in children. BMC Microbiol 10, 63.

12. Cinova J, De Palma G, Stepankova R, et al. (2011) Role of intestinal bacteria in gliadin-induced changes in intestinal mucosa: study in germ-free rats. PLoS One 6, e16169.

13. Sánchez E, Laparra JM \& Sanz Y (2012) Discerning the role of Bacteroides fragilis in celiac disease pathogenesis. Appl Environ Microbiol 78, 6507-6515.

14. De Palma G, Kamanova J, Cinova J, et al. (2012) Modulation of phenotypic and functional maturation of dendritic cells by intestinal bacteria and gliadin: relevance for celiac disease. J Leukocyte Biol 92, 1043-1054.

15. Laparra JM \& Sanz Y (2010) Bifidobacteria inhibit the inflammatory response induced by gliadins in intestinal epithelial cells via modifications of toxic peptide generation during digestion. J Cell Biochem 109, 801-807.

16. Olivares M, Laparra M \& Sanz Y (2011) Influence of Bifidobacterium longum CECT 7347 and gliadin peptides on intestinal epithelial cell proteome. J Agric Food Chem 59, 7666-7671.

17. Laparra JM, Olivares M, Gallina O, et al. (2012) Bifidobacterium longum CECT 7347 modulates immune responses in a gliadin-induced enteropathy animal model. PLoS One 7, e30744.

18. Agardh D, Lynch K, Brundin C, et al. (2006) Reduction of tissue transglutaminase autoantibody levels by glutenfree diet is associated with changes in subsets of peripheral blood lymphocytes in children with newly diagnosed coeliac disease. Clin Exp Immunol 144, 67-75.

19. Cseh Á, Vásárhelyi B, Szalay B, et al. (2011) Immune phenotype of children with newly diagnosed and gluten-free diettreated celiac disease. Dig Dis Sci 56, 792-798.

20. Cosnes J, Cellier C, Viola S, et al. (2008) Incidence of autoimmune diseases in celiac disease: protective effect of the gluten-free diet. Clin Gastroenterol Hepatol 6, 753-758.

21. Malandrino N, Capristo E, Farnetti S, et al. (2008) Metabolic and nutritional features in adult celiac patients. Dig Dis 26, $128-133$.

22. Roos S, Wilhelmsson S \& Hallert C (2011) Swedish women with coeliac disease in remission use more health care services than other women: a controlled study. Scand J Gastroenterol 46, 13-19.

23. Turco R, Boccia G, Miele E, et al. (2011) The association of coeliac disease in childhood with functional gastrointestinal disorders: a prospective study in patients fulfilling Rome III criteria. Aliment Pharmacol Ther 7, 783-789.

24. Paavola A, Kurppa K, Ukkola A, et al. (2012) Gastrointestinal symptoms and quality of life in screen-detected celiac disease. Dig Liver Dis 10, 814-818.

25. Di Sabatino A, Bertrandi E, Casadei Maldini M, et al. (1998) Phenotyping of peripheral blood lymphocytes in adult celiac disease. Immunology 95, 572-576.

26. Rubio-Tapia A, Barton SH, Rosenblatt JE, et al. (2009) Prevalence of small intestine bacterial overgrowth diagnosed by quantitative culture of intestinal aspirate in celiac disease. J Clin Gastroenterol 43, 157-161.

27. Sanz Y (2009) Novel perspectives in celiac disease therapy. Mini-Rev Med Chem 9, 359-367.
28. Farran A, Zamora R \& Cervera P (2004) Tablas de composición de alimentos CESNID (CESNID Food Composition Tables), 2nd ed. Barcelona: McGraw-Hill/Interamericana

29. Yu Y, Lee C, Kim J, et al. (2005) Group-specific primer and probe sets to detect methanogenic communities using quantitative real-time polymerase chain reaction. Biotechnol Bioeng 89, 670-679

30. Birnboim HC \& Doly J (1979) A rapid alkaline extraction procedure for screening recombinant plasmid DNA. Nucleic Acids Res 7, 1513-1523.

31. Radlović N, Mladenović M, Leković Z, et al. (2009) Effect of gluten-free diet on the growth and nutritional status of children with coeliac disease. Srp Arb Celok Lek 137, 632-637.

32. Damen GM, Boersma B, Wit JM, et al. (1994) Catch-up growth in 60 children with celiac disease. J Pediatr Gastroenterol Nutr 19, 394-400.

33. Taavela J, Kurppa K, Collin P, et al. (2013) Degree of damage to the small bowel and serum antibody titers correlate with clinical presentation of patients with celiac disease. Clin Gastroenterol Hepatol 11, 166-171.

34. Myśliwiec M, Balcerska A, Zorena K, et al. (2008) Immunologic and biochemical factors of coincident celiac disease and type 1 diabetes mellitus in children. Pediatr Res $\mathbf{6 4}$, 677-681.

35. Street ME, Volta C, Ziveri MA, et al. (2008) Changes and relationships of IGFS and IGFBPS and cytokines in coeliac disease at diagnosis and on gluten-free diet. Clin Endocrinol (Oxf) 68, 22-28.

36. Fasano A \& Shea-Donohue T (2005) Mechanisms of disease: the role of intestinal barrier function in the pathogenesis of gastrointestinal autoimmune diseases. Nat Clin Pract Gastroenterol Hepatol 2, 416-422.

37. Bayardo M, Punzi F, Bondar C, et al. (2012) Transglutaminase 2 expression is enhanced synergistically by interferon- $\gamma$ and tumour necrosis factor- $\alpha$ in human small intestine. Clin Exp Immunol 168, 95-104.

38. Rabizadeh S, Rhee KJ, Wu S, et al. (2007) Enterotoxigenic Bacteroides fragilis: a potential instigator of colitis. Inflamm Bowel Dis 13, 1475-1483.

39. De Palma G, Nadal I, Collado MC, et al. (2009) Effects of a gluten-free diet on gut microbiota and immune function in healthy adult human subjects. Br J Nutr 102, 1154-1160.

40. Scott KP, Martin JC, Duncan SH, et al. (2013) Prebiotic stimulation of human colonic butyrate-producing bacteria and bifidobacteria, in vitro. FEMS Microbiol Ecol 87, 30-40.

41. Peterson DA, McNulty NP, Guruge JL, et al. (2007) IgA response to symbiotic bacteria as a mediator of gut homeostasis. Cell Host Microbe 2, 328-339.

42. Kawamoto S, Tran TH, Maruya M, et al. (2012) The inhibitory receptor PD-1 regulates IgA selection and bacterial composition in the gut. Science 336, 485-489.

43. Delcenserie V, Martel D, Lamoureux M, et al. (2008) Immunomodulatory effects of probiotics in the intestinal tract. Curr Issues Mol Biol 10, 37-54.

44. Smecuol E, Hwang HJ, Sugai E, et al. (2013) Exploratory, randomized, double-blind, placebo-controlled study on the effects of Bifidobacterium infantis natren life start strain super strain in active celiac disease. J Clin Gastrooenterol 47, 139-147. 